J. Korean Math. Soc. 52 (2015), No. 1, pp. 81-96

http://dx.doi.org/10.4134/JKMS.2015.52.1.081

\title{
FINITE GROUPS WHOSE INTERSECTION GRAPHS ARE PLANAR
}

\author{
SelÇUK Kayacan and Ergün Yaraneri
}

\begin{abstract}
The intersection graph of a group $G$ is an undirected graph without loops and multiple edges defined as follows: the vertex set is the set of all proper non-trivial subgroups of $G$, and there is an edge between two distinct vertices $H$ and $K$ if and only if $H \cap K \neq 1$ where 1 denotes the trivial subgroup of $G$. In this paper we characterize all finite groups whose intersection graphs are planar. Our methods are elementary. Among the graphs similar to the intersection graphs, we may count the subgroup lattice and the subgroup graph of a group, each of whose planarity was already considered before in $[2,10,11,12]$.
\end{abstract}

\section{Introduction and preliminaries}

A graph is called planar if it can be drawn on the plane in such a way that its edges intersect only at their endpoints. There are interesting graphs constructed from algebraic objects such as the subgroup lattice and the subgroup graph of a group. Planarity of the subgroup lattice and the subgroup graph of a group were studied by Bohanon and Reid in [2] and by Schmidt in $[10,11]$ and by Starr and Turner III in [12], and planarity of the intersection graph of a module over any ring was studied in [13].

Here we study planarity of the intersection graph of a finite group. Let $G$ be a group. By the intersection graph of $G$ we mean an undirected graph without loops and multiple edges defined as follows: the vertex set is the set of all proper non-trivial subgroups of $G$, and there is an edge between two distinct vertices $H$ and $K$ if and only if $H \cap K \neq 1$ where 1 denotes the trivial subgroup of $G$.

We call a group planar if its intersection graph is planar. For any natural numbers $m$ and $n$, we use $C_{n}$ to denote a cyclic group of order $n$, and we write $m \mid n$ to mean that $m$ divides $n$. Our main result is:

Received March 15, 2014; Revised September 27, 2014.

2010 Mathematics Subject Classification. Primary 20D99; Secondary 20D15, 20D25, $05 \mathrm{C} 25$.

Key words and phrases. finite groups, subgroup, intersection graph, planar. 
Theorem. A finite group is planar if and only if it is isomorphic to the one of the following groups:

(1) $C_{p q r}, C_{p^{2} q}, C_{p q}, C_{p^{i}}$, where $p, q, r$ are distinct primes and $0 \leq i \leq 5$.

(2) $C_{4} \times C_{2}, C_{p} \times C_{p}, C_{2} \times C_{2} \times C_{p}(p \neq 2)$, where $p$ is a prime.

(3) The dihedral group $D_{8}$ of order 8 , the quaternion group $Q_{8}$ of order 8 .

(4) The semidirect products $C_{q} \rtimes C_{p^{2}}$ with $p^{2} \mid q-1,\left(C_{p} \times C_{p}\right) \rtimes C_{q}$ with $q \mid p+1$, where $p, q$ are distinct primes. Presentations and the subgroup structures of these groups are given in Lemma 4.6.

(5) The semidirect product $\left(C_{p} \times C_{p}\right) \rtimes C_{q^{2}}$ with $q^{2} \mid p+1$, where $p, q$ are distinct primes. A presentation and the subgroup structure of this group are given in Lemma 4.7.

(6) The semidirect product $C_{r} \rtimes C_{p q}$ with $p q \mid r-1$, where $p, q, r$ are distinct primes. A presentation and the subgroup structure of this group are given in Lemma 4.8 .

(7) The semidirect product $C_{p} \rtimes C_{q}$ with $q \mid p-1$, where $p>q$ are distinct primes. A presentation and the subgroup structure of this group are given in Lemma 4.10.

Notice that in this setting graphs with empty vertex set are allowed. In the theorem above, up to isomorphism, the first item lists the finite cyclic planar groups, the second item lists the finite non-cyclic abelian planar groups, the third item lists the finite non-abelian nilpotent planar groups, and the remaining items list the finite non-nilpotent solvable planar groups. There are no finite non-solvable planar groups.

It may be interesting to study connections between the subgroup lattice and the intersection graph of a group. It is clear that the subgroup lattice determines the intersection graph, but not conversely. Moreover, comparing our main result with the main results of $[2,11]$ we see that there are groups whose subgroup lattices are planar but the intersection graphs are not planar, and vice versa.

Our methods are elementary. For the planarity of graphs we use the Kuratowski's theorem stating that a finite graph is planar if and only if it does not contain a subdivision of either the complete graph $K_{5}$ or the complete bipartite graph $K_{3,3}$. The complete graph $K_{n}$ is a simple undirected graph with $n$ vertices in which every pair of distinct vertices is connected by a unique edge. The complete bipartite graph $K_{m, n}$ is a simple undirected graph with $m+n$ vertices and with two disjoint sets $V_{m}$ and $V_{n}$ containing exactly $m$ and $n$ vertices such that every vertex of $V_{n}$ is connected to every vertex of $V_{m}$. To study algebraic properties of the intersection graphs we use elementary group theory such as Sylow's theorem (see [5, p. 7]) and its generalization to solvable groups, namely Hall's theorem (see [5, p. 231]). We will not use any advanced result, such as results related to the classification of minimal simple groups and the odd order theorem. Below we summarize most of the results we use. For instance, we use the product formula in the third part many times without stating it explicitly. 
All the unexplained notations are standard and may be found in, for instance, $[9]$.

Remark 1.1. (1) (see $\left[9\right.$, p. 76]) Let $G$ be a group of order $p^{n}$ where $p$ is a prime. For any natural number $k$ with $k<n$, the number of subgroups of $G$ of order $p^{k}$ is $\equiv 1(\bmod p)$.

(2) (see $[9$, p. 81]) Let $P$ be a Sylow $p$-subgroup of a finite group $G$. Then, $N_{G}(H)=H$ for any subgroup $H$ with $N_{G}(P) \leq H \leq G$.

(3) (see [9, p. 30]) $|X Y||X \cap Y|=|X||Y|$ for any two subgroups $X$ and $Y$ of a finite group.

(4) (see [5, p. 131]) (Burnside $p^{m} q^{n}$ Theorem) Any group of order $p^{m} q^{n}$ is solvable where $p, q$ are prime numbers and $m, n$ are natural numbers.

(5) (see [9, p. 196]) (Burnside Normal Complement Theorem) Let $G$ be a finite group and $P$ be a Sylow $p$-subgroup of $G$. If $P$ is contained in the center of its normalizer $N_{G}(P)$ in $G$, then there is a normal subgroup $Q$ of $G$ such that $P \cap Q=1$ and $G=P Q$.

(6) (see [9, p. 197]) (Hölder's Theorem) Any finite group of square free order is solvable.

We would like to thank to anonymous referee who pointed out that, independently and using different methods, H. Ahmedi and B. Taeri have obtained the same results ("Planarity of the intersection graph of subgroups of a finite group", to appear).

\section{Abelian groups}

Modules over any ring whose intersection graphs are planar were already characterized in [13], from which the following result follows. Notice that if $H \leq G$ and $G$ is planar, then $H$ is also planar. By using this simple remark and the fundamental theorem of finite abelian groups (see [9, p. 128]), we may easily justify the following result, whose easy proof is omitted.

Proposition 2.1. A finite abelian group $G$ is planar if and only if it is isomorphic to one of the following groups:

$$
\begin{array}{cccc}
C_{p^{i}}(0 \leq i \leq 5), & C_{p^{2}} \times C_{q}, & C_{p} \times C_{q}, & C_{4} \times C_{2}, \\
C_{p} \times C_{p}, & C_{p} \times C_{q} \times C_{r}, & C_{2} \times C_{2} \times C_{p}(p \neq 2)
\end{array}
$$

where $p, q$ and $r$ are distinct primes.

\section{Nilpotent groups}

In this section we determine nilpotent groups which are planar. We first deal with $p$-groups.

Lemma 3.1. Let $p$ be a prime number and $G$ be a non-cyclic group of order $p^{4}$. Then $G$ is not planar. 
Proof. As a finite group having exactly one maximal subgroup must be cyclic, it follows from Remark 1.1 that there are at least three maximal subgroups of $G$, say $X_{1}, X_{2}$ and $X_{3}$. Since $G$ is a $p$-group of order $p^{4}$, each $X_{i}$ is of order $p^{3}$ and the product of any two of them is $G$. Employing the product formula, $Y=X_{1} \cap X_{2}$ is of order $p^{2}$ and it intersects $X_{3}$ non-trivially. Let $Z$ be a non-trivial subgroup of $X_{3} \cap Y$ of order $p$ (note that the order of $X_{3} \cap Y$ is either $p$ or $p^{2}$ ). Now, $X_{1}, X_{2}, X_{3}, Y$ and $Z$ form a $K_{5}$ in the intersection graph of $G$, so that $G$ is not planar.

Lemma 3.2. Let $p$ be an odd prime and $G$ be a non-cyclic group of order $p^{3}$. Then $G$ is not planar.

Proof. Since $p>2$, arguing as in the proof of Lemma 3.1, we first conclude that there are at least four maximal subgroups of $G$, say $X_{1}, X_{2}, X_{3}$ and $X_{4}$, of order $p^{2}$. Assume that $Y=X_{1} \cap X_{2} \cap X_{3} \cap X_{4}$ is non-trivial, then this group together with $X_{1}, X_{2}, X_{3}$ and $X_{4}$ form a $K_{5}$ in the intersection graph. Now let us assume that $Y$ is trivial. In this case $\Phi(G)=1$ where $\Phi(G)$ denotes the Frattini subgroup of $G$. Since $G$ is a p-group, $G / \Phi(G)$ is elementary abelian. Thus, if $\Phi(G)=1$ and $|G|=p^{3}$, then $G \cong G / \Phi(G) \cong C_{p} \times C_{p} \times C_{p}$ which is not planar, because $C_{p} \times C_{p} \times C_{p}$ is not listed in Proposition 2.1.

Up to isomorphism, there are exactly 5 distinct groups of order 8 and only two of them, namely $D_{8}$ (dihedral group of order 8) and $Q_{8}$ (quaternion group), are non-abelian. Both groups, whose intersection graphs are given in Figure 1, are planar.

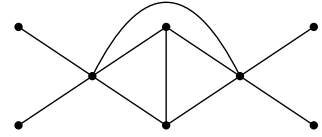

(A) $D_{8}$

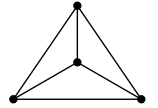

(B) $Q_{8}$

FIGURE 1. Non-abelian planar nilpotent groups.

It is clear that if $H$ is a proper subgroup of $G$ and the intersection graph of $H$ contains $K_{4}$, then $G$ cannot be planar, because there would be a $K_{5}$ in the graph. With this simple remark we have:

Proposition 3.3. A finite non-abelian nilpotent group is planar if and only if it is isomorphic to $D_{8}$ or $Q_{8}$.

Proof. Suppose that $G$ is a finite non-abelian nilpotent group which is planar. Since a nilpotent group is the direct product of its Sylow subgroups, there must be a non-abelian Sylow subgroup $S$ of $G$. Let $|S|=p^{\alpha}$ for some prime $p$ and natural number $\alpha$. Since $S$ is non-abelian, $\alpha \geq 3$. As $S$ must be planar, it follows from Lemma 3.1 and Lemma 3.2 that $\alpha=3$ and $p=2$, which means 
$S$ must be isomorphic to $D_{8}$ or $Q_{8}$. In both cases the intersection graph of $S$ contains $K_{4}$. Therefore $S$ cannot be a proper subgroup of $G$, and so $G=S$.

\section{Solvable groups}

As a subgroup of a planar group is planar, the following lemma is an easy consequence of Propositions 2.1 and 3.3.

Lemma 4.1. Let $G$ be a finite planar group of order $n=p_{1}^{\alpha_{1}} p_{2}^{\alpha_{2}} \cdots p_{k}^{\alpha_{k}}$ where $k>2$ and $p_{i}$ are distinct prime numbers. Then $\alpha_{j}<5$ for any $j$. Moreover, if $\alpha_{j}=3$ or $\alpha_{j}=4$ for some $j$, then any Sylow $p_{j}$-subgroup of $G$ is cyclic.

Proof. There is only one planar group of order $p^{5}$, namely $C_{p^{5}}$, and only one planar group of order $p^{4}$, namely $C_{p^{4}}$, and four planar groups of order $p^{3}$, namely $C_{p^{3}}, C_{4} \times C_{2}, D_{8}$ and $Q_{8}$ (see Propositions 2.1 and 3.3). But, each of $C_{p^{5}}, C_{4} \times C_{2}, D_{8}$ and $Q_{8}$ contains $K_{4}$ in its intersection graph.

A finite solvable group is a group with a composition series whose factor groups are of prime order. This means that if $G$ is a planar solvable group of order $p_{1}^{\alpha_{1}} p_{2}^{\alpha_{2}} \cdots p_{k}^{\alpha_{k}}$ where $p_{i}$ are distinct prime numbers, then $\alpha_{1}+\alpha_{2}+\cdots+$ $\alpha_{k}<6$; otherwise, there must be a chain of five proper non-trivial subgroups forming a $K_{5}$ in the intersection graph. Hence, for a finite solvable group $G$ there are finitely many cases that must be examined, and these cases are given in Table 1, whose first row consist of $p$-groups and they are already classified.

TABle 1

$$
\begin{array}{l|l|l|l}
|G|=p^{5} & |G|=p^{4} & |G|=p^{3} & |G|=p^{2} \\
|G|=p^{4} q & |G|=p^{3} q & |G|=p^{2} q & |G|=p q \\
|G|=p^{3} q^{2} & |G|=p^{2} q^{2} & |G|=p q r & \\
|G|=p^{3} q r & |G|=p^{2} q r & & \\
|G|=p^{2} q^{2} r & |G|=p q r t & & \\
|G|=p^{2} q r t & & & \\
|G|=p q r t u & &
\end{array}
$$

Note that the groups in Lemmas 4.2-4.3 and 4.6-4.8 are all solvable by the virtue of Burnside's and Hölder's theorems (see Remark 1.1).

We say that non-trivial subgroups $H_{1}, H_{2}, \ldots, H_{n}$ of a group $G$ are mutually intersecting if $H_{i} \cap H_{j} \neq 1$ for any $i$ and $j$.

We first eliminate groups of order $p^{3} q$ and of order $p^{4} q$ as non-planar groups.

Lemma 4.2. If $G$ is a group of order $p^{3} q$ or $p^{4} q$ where $p$ and $q$ are distinct prime numbers, then $G$ is not planar.

Proof. We prove the assertion for groups of order $p^{3} q$. Similar arguments apply for groups of order $p^{4} q$. Let $P$ be a Sylow $p$-subgroup of $G$ and let $Q$ be a Sylow $q$-subgroup of $G$. By Lemma 4.1 we see that $P$ is cyclic, otherwise $G$ is not 
planar. Take a chain $A_{1}<A_{2}<P$ where $\left|A_{1}\right|=p$ and $\left|A_{2}\right|=p^{2}$. We have three cases to analyze: in the first case $P$ is normal in $G$; in the second case $Q$ is normal in $G$; and in the third case both $P$ and $Q$ are not normal in $G$.

Case $I$ : Assume that $P$ is normal in $G$. As any subgroup of a normal cyclic subgroup is also a normal subgroup, each $A_{i}$ is normal in $G$, implying that the products $A_{i} Q$ are subgroups of $G$. It is now clear that the five subgroups $A_{1}, A_{2}, P, A_{1} Q, A_{2} Q$ are mutually distinct and each of them contains $A_{1}$. Consequently, the graph of $G$ contains $K_{5}$, and so $G$ is not planar.

Case II: Assume that $Q$ is normal in $G$. In this case the products $A_{i} Q$ are subgroups of $G$. So, as in the first case, $A_{1}, A_{2}, P, A_{1} Q$ and $A_{2} Q$ form a $K_{5}$ in the graph of $G$, and so $G$ is not planar.

Case III: Assume that both of $P$ and $Q$ are not normal in $G$. Let $N$ be a minimal normal subgroup of $G$. Since $G$ is solvable, $N$ is an elementary abelian $r$-group for some prime $r$ (that is, a direct product of cyclic groups $C_{r}$ ). As $Q$ is not normal, $r=p$. Therefore, $N$ is an elementary abelian $p$-group inside the cyclic $p$-group $P$. This shows that $N \cong C_{p}$. Now take a subgroup $T$ such that $N<T<P$ where $|T|=p^{2}$. As each Sylow $p$-subgroup of $G$ contains $N$, we see that each Sylow $p$-subgroup of $G$ intersects $T$. Consequently, all the Sylow $p$-subgroups together with the subgroups $N$ and $T$ are mutually intersecting and mutually distinct. As $P$ is not normal, there are at least $p+1$ Sylow $p$-subgroups. Therefore, in the above we have at least $p+3$ mutually distinct and mutually intersecting subgroups. As $p+3 \geq 5, G$ cannot be planar.

Lemma 4.3. Let $G$ be a group of order $p^{3} q^{2}$ where $p$ and $q$ are distinct prime numbers. Then $G$ is not planar.

Proof. Since $G$ is solvable, there must be a (normal) subgroup $H$ of order either $p^{2} q^{2}$ or $p^{3} q$. By Lemma 4.2 we eliminate the latter case. Then $H$ has a subgroup $K$ of order either $p^{2} q$ or $p q^{2}$. Let $X$ be a subgroup of $K$ of order $p$, and let $P$ be a Sylow $p$-subgroup of $G$ containing $X$. As $|X|=p$ and $|P|=p^{3}$, we may choose a subgroup $Y$ of $G$ such that $X<Y<P$ with $|Y|=p^{2}$. Then, $H, K, X, Y, P$ form a $K_{5}$ in the intersection graph of $G$.

Let $G$ be a finite group and let $N$ be a non-trivial normal subgroup of $G$. If $G / N$ has at least five proper subgroups, then by the correspondence theorem $G$ has at least five proper subgroups all containing $N$ and these subgroups form a $K_{5}$ in the intersection graph of $G$. The groups having exactly $m$ subgroups where $m \leq 6$ are classified in [8].

Proposition 4.4 (see [8]). A non-abelian group has at least 6 subgroups.

It follows easily from the previous result that the center $Z(G)$ of any nonnilpotent planar group $G$ is trivial. Another immediate consequence of the classification in [8] is the following. 
Lemma 4.5. Let $G$ be a finite planar group and let $N$ be a non-trivial normal subgroup of $G$. Then $G / N$ is abelian. Moreover, letting $n_{s}$ be the number of proper non-trivial subgroups of $G / N$, the following occur:

(1) $n_{s}=0 \Rightarrow G / N \cong C_{p}$

(2) $n_{s}=1 \Rightarrow G / N \cong C_{p^{2}}$

(3) $n_{s}=2 \Rightarrow G / N \cong C_{p^{3}}$ or $G / N \cong C_{p q}$

(4) $n_{s}=3 \Rightarrow G / N \cong C_{p^{4}}$ or $G / N \cong C_{2} \times C_{2}$

for some distinct prime numbers $p$ and $q$.

We use the above result to reduce the number of possible cases for the order of a finite planar solvable group. Let $G$ be a finite planar solvable group, and let $N$ be a minimal normal subgroup of $G$. Then $N$ must be a planar elementary abelian $s$-group where $s$ is a prime number. It follows from Proposition 2.1 that $N$ is isomorphic to $C_{s}$ or $C_{s} \times C_{s}$. Moreover, $G / N$ must be isomorphic to one of the groups described in Lemma 4.5. Therefore, the solvable groups of order $p^{3} q r, p^{2} q^{2} r$, pqrt, $p^{2} q r t$ and pqrtu given in Table 1 cannot be planar.

Lemma 4.6. Let $G$ be a non-nilpotent group of order $p^{2} q$ where $p$ and $q$ are distinct prime numbers. Then, $G$ is planar if and only if it is isomorphic to one of the following groups:

(1)

$$
C_{q} \rtimes_{\alpha} C_{p^{2}}=\left\langle a, b \mid a^{q}=b^{p^{2}}=1, b a b^{-1}=a^{\alpha}\right\rangle
$$

where $p^{2}$ divides $q-1$ and $\alpha$ is any integer not divisible by $q$ whose order in the unit group $\mathbb{Z}_{q}^{*}$ of $\mathbb{Z}_{q}$ is $p^{2}$. (Moreover, such a group has exactly $q$ subgroups of order $p^{2}$ which are all cyclic and mutually nonintersecting, and has exactly 1 subgroup of order $q$, and has exactly 1 (2) subgroup of order $p q$, and has exactly $q$ subgroups of order $p$ ).

$\left(C_{p} \times C_{p}\right) \rtimes_{\beta} C_{q}=\left\langle a, b, c \mid a^{p}=b^{p}=c^{q}=1, a b=b a, c a c^{-1}=b, c b c^{-1}=a^{-1} b^{\beta}\right\rangle$ where $q$ divides $p+1$ and $\beta$ is any integer such that the matrix $\theta=$ $\left[\begin{array}{cc}0 & -1 \\ 1 & \beta\end{array}\right]$ has order $q$ in the group $G L\left(2, \mathbb{Z}_{p}\right)$ and such that $\theta$ has no eigenvalue in $\mathbb{Z}_{p}$. (Moreover, such a group has exactly 1 subgroup of order $p^{2}$ which is elementary abelian, and has exactly $p^{2}$ subgroups of order $q$, and has exactly $p+1$ subgroups of order $p$, and has no subgroup of order $p q$. )

Proof. Let $G$ be a non-nilpotent group of order $p^{2} q$. Let $P$ be a Sylow $p$ subgroup of $G$ and $Q$ be a Sylow $q$-subgroup of $G$. We separate the proof into two parts. In the first case we assume that $P$ is not normal in $G$ and in the second case we assume that $P$ is normal in $G$.

Case $I$ : Assume that $P$ is not normal: As $P$ is a maximal subgroup and as it has order $p^{2}$, we see that the center of $N_{G}(P)$ is $P$, from which we conclude by using the Burnside Normal Complement Theorem (see Remark 1.1) 
that $Q$ is normal in $G$. Moreover, the Sylow theorems imply that $G$ has $q$ Sylow $p$-subgroups $P_{1}, \ldots, P_{q}$ and $q \equiv 1(\bmod p)$. As $|P|=p^{2}$, there are two possibilities: $P \cong C_{p} \times C_{p}$ or $P \cong C_{p^{2}}$.

Case $I$ (a): Assume that $P \cong C_{p} \times C_{p}$ : As $G=P Q$ and $Q$ is normal in $G$, we see that $G / Q \cong P$. Now $P$ has $p+1$ subgroups of order $p$, and hence by the correspondence theorem $G$ has $p+1$ subgroups $R_{1}, R_{2}, \ldots, R_{p+1}$ of order $p q$ (all of which contain $Q$ ). As $\left|P_{i}\right|\left|R_{j}\right|=p^{2} p q>|G|$, we see that $P_{i} \cap R_{j} \neq 1$. Since both of $q$ and $p+1$ are greater than or equal to 3 , we see that $P_{1}, P_{2}, P_{3}$ and $R_{1}, R_{2}, R_{3}$ form a $K_{3,3}$ in the intersection graph of $G$. Hence, $G$ is not planar in this case.

Case $I(\mathrm{~b})$ : Assume that $P \cong C_{p^{2}}$ :

Case $I(\mathrm{~b})(\mathrm{i})$ : Assume that $P_{i} \cap P_{j} \neq 1$ for some distinct $i$ and $j$ : Let $X=P_{i} \cap P_{j}$. Then $N_{G}(X)$ contains both of $P_{i}$ and $P_{j}$, implying that $X$ is a normal subgroup of $G$ of order $p$. Therefore, $X$ is in every Sylow $p$-subgroups of $G$. Hence, $P_{1}, P_{2}, \ldots, P_{q}$ and $X$ and $Q X$ form a $K_{q+2}$ in the intersection graph of $G$. Note that as $q \equiv 1(\bmod p), q+2 \geq 5$. So, $G$ is not planar in this case.

Case I (b)(ii): Assume the contrary of the previous case: That is, we assume that the intersection of any two distinct Sylow $p$-subgroups is trivial. As $G / Q \cong P$ and $P$ is cyclic, $G / Q$ has a unique subgroup of order $p$. From the correspondence theorem $G$ has a unique subgroup of order $p q$. So, in this case, it is clear that $G$ is planar, and its intersection graph is given in Figure 2. To write a presentation of $G$ let $a$ be a generator of $Q$ and $b$ be a generator of $P$. Then $b a b^{-1}=a^{\alpha}$ for some integer $\alpha$. For any natural number $k$, it is easy to see that $b^{k} a b^{-k}=a^{\alpha^{k}}$. This shows that $\alpha^{p^{2}} \equiv 1(\bmod q)$. Moreover, $\alpha^{p} \not \equiv 1$ $(\bmod q)$, otherwise the intersection of any two Sylow $p$-subgroups of $G$ is not trivial. Conversely, it is clear that any group with the given presentation has the stated subgroup structure.

Case II: Assume that $P$ is normal in $G$. As $G$ is not nilpotent, $Q$ cannot be normal in $G$. We have two possibilities either there is a subgroup of $G$ of order $p q$ or there is no such subgroup.

Case II (a): Assume that there is a subgroup of $G$ of order $p q$ :

Case II (a)(i): Assume that there is a normal subgroup of $G$ of order $p q$, say $Y$. Then Sylow $q$-subgroups of $Y$ and $G$ are the same, implying that $Y$ and hence $G$ has $p$ Sylow $q$-subgroups $Q_{1}, Q_{2}, \ldots, Q_{p}$, and $p \equiv 1(\bmod q)$. Note that the normalizers $N_{G}\left(Q_{1}\right), N_{G}\left(Q_{2}\right), \ldots, N_{G}\left(Q_{p}\right)$ must be mutually distinct, because each $N_{G}\left(Q_{i}\right)$ has a unique Sylow $q$-subgroup which is $Q_{i}$. Moreover, they all have order $p q$. As the normalizer of a Sylow subgroup is self normalizing (see the second part of Remark 1.1), each $N_{G}\left(Q_{i}\right)$ is not normal in $G$. Therefore, we see that the $p+2$ subgroups $P, Y, N_{G}\left(Q_{1}\right), N_{G}\left(Q_{2}\right), \ldots, N_{G}\left(Q_{p}\right)$ are mutually distinct and intersecting, forming a $K_{p+2}$ in the intersection graph of $G$. Since $p+2 \geq 5($ because $p \equiv 1(\bmod q)), G$ is not planar. 
Case II (a)(ii): Assume that there is a non-normal subgroup of $G$ of order $p q$, say $Z$. Its index $p$ cannot be the smallest prime dividing the order of $G$. Hence, $p>q$. Let $U$ be a Sylow $p$-subgroup of $Z$. Then $U$ must be normal in $Z$. Note that $U$ is contained in $P$ (because $P$ is normal in $G$ ) and that $U$ is normal in $P$ (because $P$ is abelian). Therefore, $U$ is normal in $G$. It follows from Proposition 4.4 and the explanation given before it that if the quotient group $G / U$ is not abelian, then $G$ is not planar. On the other hand, if $G / U$ is abelian, then the correspondence theorem implies that $G$ has a normal subgroup of order $p q$. We know from the previous subcase that in this case $G$ is not planar.

Case II (b): Assume that there is no subgroup of $G$ of order $p q$ : In this case it is clear that $G$ is planar. Moreover, $P$ cannot be cyclic. Otherwise, its unique subgroup $T$ of order $p$ will be a normal subgroup of $G$, implying that the quotient group $G / T$ will have a subgroup of order $q$, and hence $G$ will have a subgroup of order $p q$. Therefore, $P \cong C_{p} \times C_{p}$. As $G$ has no subgroup of order $p q$, the normalizer of a Sylow $q$-subgroup of $G$ must have index $p^{2}$. The subgroup $Q$ acts by conjugation on the set of all subgroups of $G$ of order $p$. As $G$ has no subgroup of order $p q$, this action has no fixed point, implying that $q$ divides $p+1$.

To write a presentation of $G$, let $a$ be an element of $P$ of order $p$ and let $c$ be a generator of $Q$. As $G$ has no subgroup of order $p q$, the elements $a$ and $c a c^{-1}$ must generate $P$. Letting $b:=c a c^{-1}$, it is enough to determine $c b c^{-1}$ in terms of $a$ and $b$. Now $c b c^{-1}=a^{\gamma} b^{\beta}$ for some integers $\gamma$ and $\beta$. Conjugation by $c$ induces an invertible linear operator $f$ on the vector space $P$ over the field $\mathbb{Z}_{p}$ and the matrix of $f$ with respect to the basis $\{a, b\}$ of $P$ is $\theta=\left[\begin{array}{ll}0 & \gamma \\ 1 & \beta\end{array}\right]$.

Note that $G$ has no subgroup of order $p q$ if and only if $f$ has no eigenvalue in $\mathbb{Z}_{p}$ : Indeed, if $1 \neq s \in P$ is an eigenvector of $f$ corresponding to an eigenvalue $\lambda \in \mathbb{Z}_{p}$, then $f(s)=s^{\lambda}$, implying that $\langle c\rangle\langle s\rangle$ is a subgroup of $G$ (because $\langle c\rangle\langle s\rangle=\langle s\rangle\langle c\rangle)$ of order $p q$. Conversely, if $G$ has a subgroup of order $p q$, then conjugating it by an element of $G$ we see that there is a subgroup $H$ of $G$ of order $p q$ which contains $Q$. Therefore, $H=\langle t\rangle\langle c\rangle$ for some $1 \neq t \in P$. As $H$ is a subgroup, $c t=t^{m} c^{n}$ for some integers $m$ and $n$. Then $t^{-m} f(t)=c^{n-1} \in$ $P \cap Q=1$, implying that $f(t)=t^{m}$.

As the order of $c$ is prime $q$, the order of $\theta$ in $G L\left(2, \mathbb{Z}_{p}\right)$ must be $q$. Considering the determinants we see from the equation $\theta^{q}=I$ that the possibilities for the order of $-\gamma$ in $\mathbb{Z}_{p}^{*}$ is 1 or $q$. Suppose for a moment that the order of $-\gamma$ is $q$. Then $q$ divides $p-1$, implying that $q=2$ (because we already know that $q$ divides $p+1)$. But then $\theta^{2}=I$ implies that $\theta=\left[\begin{array}{ll}0 & 1 \\ 1 & 0\end{array}\right]$, which has an eigenvalue in $\mathbb{Z}_{p}$. Therefore, the order of $-\gamma$ must be 1 , implying that $\gamma \equiv-1$ $(\bmod p)$.

Conversely, it is clear that any group with the given presentation has the stated subgroup structure. The intersection graph of such a group is given in Figure 2. 
The prime $q$ in the second part of the previous lemma cannot be 2 . Indeed, it is easy to see that $\theta^{2} \neq I$ where $\theta$ is the matrix in Lemma 4.6. Therefore, there is no planar group of order $2 p^{2}$ where $p$ is an odd prime.

Groups of order $p^{2} q$ were classified by Hölder (see [6], [3, p. 76], [4], or [1]). The previous lemma may also be justified by analyzing the cases described in these references.

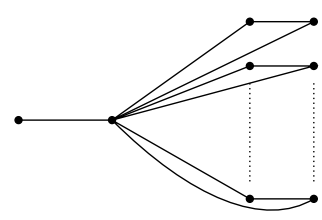

(A) $C_{q} \rtimes_{\alpha} C_{p^{2}}$

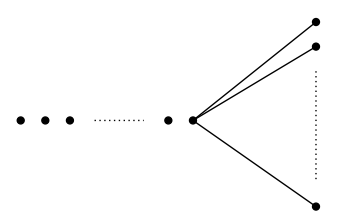

(в) $\left(C_{p} \times C_{p}\right) \rtimes_{\beta} C_{q}$

FiguRE 2. Non-nilpotent planar groups of order $p^{2} q$.

Lemma 4.7. Let $G$ be a non-nilpotent group of order $p^{2} q^{2}$ where $p>q$ are distinct prime numbers. Then, $G$ is planar if and only if it is isomorphic to

$\left(C_{p} \times C_{p}\right) \rtimes_{\beta} C_{q^{2}}=\left\langle a, b, c \mid a^{p}=b^{p}=c^{q^{2}}=1, a b=b a, c a c^{-1}=b, c b c^{-1}=a^{-1} b^{\beta}\right\rangle$ where $q^{2}$ divides $p+1$ and $\beta$ is any integer such that the matrix $\theta=\left[\begin{array}{cc}0 & -1 \\ 1 & \beta\end{array}\right]$ has order $q^{2}$ in the group $G L\left(2, \mathbb{Z}_{p}\right)$ and such that $\theta^{q}$ has no eigenvalue in $\mathbb{Z}_{p}$. (Moreover, such a group has exactly 1 subgroup of order $p^{2} q$, and has no subgroup of order $p q^{2}$, and has exactly 1 subgroup of order $p^{2}$ which is elementary abelian, and has exactly $p^{2}$ subgroups of order $q^{2}$ which are all cyclic and mutually non-intersecting, and has no subgroup of order $p q$, and has exactly $p+1$ subgroups of order $p$, and has exactly $p^{2}$ subgroups of order q.)

Proof. Assume that $G$ is planar. Let $P$ be a Sylow $p$-subgroup of $G$ and $Q$ be a Sylow $q$-subgroup of $G$. We have the following subgroup structure for $G$ :

(I) $G$ has no normal subgroup of prime order: This follows from Lemma 4.5.

(II) $P$ is normal in $G$ and $Q$ is not normal in $G$ : It is clear that the intersection of any two distinct Sylow $p$-subgroups $P_{1}$ and $P_{2}$ of $G$ is a normal subgroup of $G$ of order $p$. The normality of $P_{1} \cap P_{2}$ may be seen easily by considering the normalizer of $P_{1} \cap P_{2}$. Therefore, it follows from (I) that $P$ is normal in $G$. As $G$ is not nilpotent, $Q$ is not normal in $G$.

(III) $P \cong C_{p} \times C_{p}$ : Using Lemma 4.5 and (I) and (II) we see that $P$ is a minimal normal subgroup of $G$.

(IV) $Q \cong C_{q^{2}}$ : It follows from Lemma 4.5 that $G / P \cong C_{q^{2}}$ or $G / P \cong$ $C_{2} \times C_{2}$. In the case $G / P \cong C_{2} \times C_{2}$, there are 3 subgroups of $G / P$ of order 2. Hence, there are 3 subgroups $X_{1}, X_{2}, X_{3}$ of $G$ of order $2 p^{2}$ all of which contain $P$. Letting $Y$ be any subgroup of $P$ of order $p$, we see that the groups $X_{1}, X_{2}, X_{3}, P, Y$ form a $K_{5}$ in the intersection graph of $G$ so that $G$ is not planar. 
(V) $G$ has exactly one subgroup of order $p^{2} q$ : As $P$ is normal in $G$, any subgroup of $G$ of order divisible by $p^{2}$ contains $P$. So, the number of subgroups of $G$ of order $p^{2} q$ is equal to the number of subgroups of $Q \cong G / P$ of order $q$. The result follows because $Q$ is cyclic by (IV).

(VI) $G$ has no subgroup of order $p q^{2}$ and has no subgroup of order $p q$ : There is a unique subgroup of $G$ of order $p^{2} q$ by (V). This subgroup, say $H$, must be planar and $P$ is the unique Sylow $p$-subgroup of $H$. As $P$ is elementary abelian, $H$ must be isomorphic to the second group found in Lemma 4.6. In particular, $H$ has no normal subgroup of order $p$ and has no subgroup of order $p q$. Now, suppose for a moment that there is a subgroup $U$ of $G$ of order $p q^{2}$ or $p q$. Note that $U$ is not in $H$. As $P$ is an abelian normal subgroup of $G$, we see that $P N_{G}(U) \leq N_{G}(P \cap U)$. Considering the order of the subgroup $P N_{G}(U)$ and the uniqueness of $H$, we see that $H \leq P N_{G}(U)$. Therefore, $P \cap U$ is a normal subgroup of $H$ of order $p$, which is impossible.

(VII) $G$ has exactly $p^{2}$ subgroups of order $q^{2}$, all of which are cyclic: As $Q$ is not normal, this follows from (IV), (VI), and the Sylow theorems.

(VIII) The intersection of any two distinct subgroups of order $q^{2}$ is trivial: Otherwise the intersection is a subgroup of $G$ of order $q$ such that the order of the normalizer of the intersection is $p q^{2}$ or $p^{2} q^{2}$. It follows from (VI) and (I) that each of the two cases is impossible.

(IX) $G$ has exactly $p^{2}$ subgroups of order $q$ : This follows from (VII) and (VIII).

(X) $q^{2}$ divides $p+1: Q$ acts by conjugation on the set of all subgroups of $G$ of order $p$. Since by (VI) there is no subgroup of $G$ of order $p q^{2}$ or $p q$, the stabilizer of any subgroup of $G$ of order $p$ must be the trivial subgroup of $Q$. Therefore, each orbit has cardinality $q^{2}$.

Conversely, it is clear that any group satisfying the above properties (I)-(X) is planar, and its intersection graph is given in Figure 3. On the left the vertices represent the subgroup of order $p^{2}$ and the $p+1$ subgroups of order $p$, and on the rightmost two columns the vertices represent subgroups of order $q$ and of order $q^{2}$.

Finally, we may argue as in the proof of the second part of Lemma 4.6 to see that such a group has the given presentation. Indeed, let $a$ be an element of $P$ of order $p$ and let $c$ be a generator of $Q$. As $G$ has no subgroup of order $p q^{2}$, the elements $a$ and $b:=c a c^{-1}$ form a basis for the vector space $P$ over $\mathbb{Z}_{p}$. Now $c b c^{-1}=a^{\gamma} b^{\beta}$ for some integers $\gamma$ and $\beta$. The matrix of the conjugation on $P$ by $c$ is $\theta=\left[\begin{array}{ll}0 & \gamma \\ 1 & \beta\end{array}\right]$. The order of $\theta$ in $G L\left(2, \mathbb{Z}_{p}\right)$ must be $q^{2}$ because $c$ has order $q^{2}$ and $G$ has no subgroup of order $p q$. The order of $(-\gamma)$ in $\mathbb{Z}_{p}^{*}$ is 1 or $q$ or $q^{2}$. We see easily that the order is not $q^{2}$ (otherwise $q^{2}$ divides 2) and is not $q$ (otherwise, $q=2$ and $\gamma^{2}=1$ in $\mathbb{Z}_{p}$, and $\theta^{4}=I$ implies that $\beta=0$ in $\mathbb{Z}_{p}$, and so $\theta^{2}$ is diagonal, implying that $G$ has a subgroup of order $p q$ ). Therefore, $\gamma \equiv-1(\bmod p)$. Moreover, as $G$ has no subgroup of order $p q$ we have to assume that $\theta^{q}$ (implying that $\theta$ ) has no eigenvalue in $\mathbb{Z}_{p}$. 
The prime $q$ in the previous lemma cannot be 2 . Indeed, $\theta^{4}=I$ implies that $\theta^{2}$ is diagonal, and so $G$ has a subgroup of order $p q$, where $\theta$ is the matrix in Lemma 4.7. Therefore, there is no planar group of order $4 p^{2}$ where $p$ is an odd prime.

Groups of order $p^{2} q^{2}$ were determined by Le Vavasseur in [7]. The previous result may also be proved by analyzing the cases given there.

Lemma 4.8. Let $G$ be a non-nilpotent group of order pqr where $p<q<r$ are distinct prime numbers. Then, $G$ is planar if and only if it is isomorphic to

$$
C_{r} \rtimes_{\alpha} C_{p q}=\left\langle a, b \mid a^{r}=b^{p q}=1, b a b^{-1}=a^{\alpha}\right\rangle
$$

where $p q$ divides $r-1$ and $\alpha$ is any integer not divisible by $r$ whose order in the unit group $\mathbb{Z}_{r}^{*}$ of $\mathbb{Z}_{r}$ is pq. (Moreover, such a group has exactly 1 subgroup of order pr, and has exactly 1 subgroup of order qr, and has exactly $r$ subgroups of order pq, which are all cyclic and mutually non-intersecting, and has exactly 1 subgroup of order $r$, and has exactly $r$ subgroups of order $p$, and has exactly $r$ subgroups of order $q$ ).

Proof. The Sylow theorems imply that $G$ has a unique Sylow $r$-subgroup $R$. Assume first that $G$ is planar. We have the following subgroup structure for $G$ :

(I) $G$ has exactly 1 subgroup of order $p r$ and exactly 1 subgroup of order $q r$ : From Lemma 4.5 , we see that $G / R \cong C_{p q}$. Thus $G / R$ has exactly one subgroup of order $p$ and $q$. Since any subgroup of $G$ of order divisible by $r$ contains $R$, the result follows.

(II) $G$ has exactly $r$ subgroups of order $p q$, which are all cyclic and mutually non-intersecting: Let $X$ be a Hall subgroup of $G$ of order $p q$. By (I) there are unique subgroups of $G$ of order $p r$ and $q r$, say $Y$ and $Z$. If $X$ is normal in $G$, then $X \cap Y \cap Z=1$ so that $G$ is isomorphic to a subgroup of the cyclic group $C_{r} \times C_{q} \times C_{p}$. Therefore, $X$ is not normal in $G$. As any two Hall subgroups of a finite solvable group of the same order are conjugate [5, p. 231, Theorem 4.1], there are exactly $r$ subgroups of $G$ of order $p q$. Moreover, $X$ must be cyclic because $X \cap Y$ and $X \cap Z$ are normal subgroups of $X$ of orders $p$ and $q$ whose product is $X$. Finally, let $X_{1}$ and $X_{2}$ be two subgroups of $G$ of order $p q$ such that $X_{1} \cap X_{2} \neq 1$. Then we see that $X_{1} \cap X_{2}$ is a normal subgroup of $G$ of order $p$ or $q$. But then $X_{1} \cap X_{2}$ must be contained in each of $r$ subgroups of $G$ of order $p q$. Therefore, the intersection graph of $G$ contains $K_{r}$. As $r \geq 5$, in this case $G$ is not planar.

(III) $G$ has exactly $r$ subgroups of order $p$ and $r$ subgroups of order $q$ : As any subgroup of $G$ of order $p$ or $q$ is contained in a subgroup of $G$ of order $p q$, the result follows from (II).

(IV) $p q$ divides $r-1$ : It follows from (III) and the Sylow theorems that $r \equiv 1(\bmod p)$ and $r \equiv 1(\bmod q)$. The result follows.

Conversely, it is clear that any group satisfying the above properties (I)-(IV) is planar, and its intersection graph is given in Figure 3. The uppermost middle 
vertex represents the unique subgroup of $G$ of order $r$, and the leftmost and the rightmost vertices represent the unique subgroups of $G$ of order $p r$ and $q r$.

Finally, we may argue as in the proof of the first part of Lemma 4.6 to see that such a group has the given presentation.

Groups of order $p q r$ were classified by Hölder (see [6]). One may also analyze the cases there to prove the previous result.

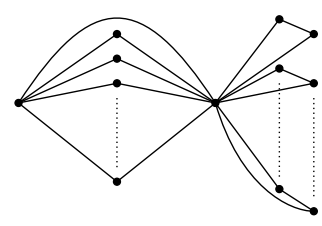

(A) $\left(C_{p} \times C_{p}\right) \rtimes_{\beta} C_{q^{2}}$

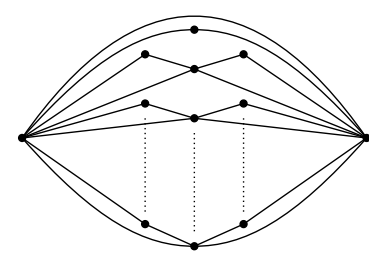

(в) $C_{r} \rtimes_{\alpha} C_{p q}$

FIGURE 3. Non-nilpotent planar groups of order $p^{2} q^{2}$ and of order $p q r$.

Lemma 4.9. Let $G$ be a non-nilpotent solvable group of order $p^{2} q r$ where $p, q$ and $r$ are distinct prime numbers. Then $G$ is not planar.

Proof. Assume for a moment that $G$ is planar. Let $P$ be a Sylow $p$-subgroup of $G$. We have the following subgroup structure for $G$ :

(I) $P$ is normal in $G$ and $P \cong C_{p} \times C_{p}$ and $G / P \cong C_{q r}$ : As any minimal normal subgroup of a finite solvable group is elementary abelian group of prime power order, the result follows from Lemma 4.5 .

(II) $G$ has exactly 1 subgroup $A$ of order $p^{2} q$ and has exactly 1 subgroup $B$ of order $p^{2} r$. Moreover, both $A$ and $B$ contain $P$ : As $P$ is normal, any subgroup of $G$ of order divisible by $p^{2}$ must contain $P$. The result follows from (I) which implies that $G / P$ has exactly 1 subgroup of order $q$ and has exactly 1 subgroup of order $r$.

(III) The intersection graph of $G$ contains $K_{3,3}$ : It follows from (I) that $P$ has exactly $p+1$ subgroups of order $p$. Take any 3 distinct subgroups of $P$ of order $p$, say $X_{1}, X_{2}, X_{3}$. Then, it is clear from (II) that the intersection of any element of the set $\left\{X_{1}, X_{2}, X_{3}\right\}$ with any element of the set $\{A, B, P\}$ is not trivial. Thus, the intersection graph of $G$ contains $K_{3,3}$.

Finally, we note that (III) contradicts the planarity of $G$.

Finally, if $G$ is a group of order $p q$ where $p>q$ are prime numbers, then any proper non-trivial subgroup of $G$ is of prime order, and so there is no edge in the intersection graph of $G$. Therefore, any such group is planar, and we have the following easy consequence of the Sylow theorems. 
Lemma 4.10. Let $G$ be a group of order $p q$ where $p>q$ are distinct primes. Then, $G$ is planar. If $G$ is non-nilpotent, then $q$ divides $p-1$ and $G$ is isomorphic to

$$
C_{p} \rtimes_{\alpha} C_{q}=\left\langle a, b \mid a^{p}=b^{q}=1, b a b^{-1}=a^{\alpha}\right\rangle
$$

where $\alpha$ is any integer not divisible by $p$ whose order in the unit group $\mathbb{Z}_{p}^{*}$ of $\mathbb{Z}_{p}$ is q. (Moreover, such a group has exactly 1 subgroup of order $p$ and has exactly p subgroups of order $q$ ).

\section{Non-solvable groups}

In this section we show that any non-solvable finite group is not planar.

Lemma 5.1. If $G$ is a finite non-solvable simple group, then $G$ is not planar.

Proof. Suppose contrarily that $G$ is a finite non-solvable simple group which is planar. Then we have:

(I) Any Sylow subgroup of $G$ is abelian: Let $P$ be a Sylow $p$-subgroup of $G$ for some prime $p$ dividing $|G|$. As $P$ is planar, it follows from Propositions 2.1 and 3.3 that $P$ is isomorphic to one of the groups $C_{p^{\alpha}}(\alpha \leq 5), C_{p} \times C_{p}, D_{8}$, $Q_{8}$. However, the intersection graph of any of the groups $C_{p^{5}}, D_{8}, Q_{8}$ contains a $K_{4}$. Therefore, $P$ must be isomorphic to one of the groups $C_{p^{\alpha}}(\alpha \leq 4)$, $C_{p} \times C_{p}$.

(II) For any non-trivial Sylow subgroup $P$ of $G$, its normalizer $N_{G}(P)$ is a non-abelian proper subgroup of $G$ : As $G$ is simple, the result follows from the Burnside Normal Complement Theorem (see Remark 1.1).

(III) If $P$ is a Sylow $p$-subgroup of $G$ for some prime $p$ dividing $|G|$, then $P$ is isomorphic to $C_{p} \times C_{p}$ or $C_{p}$. Moreover, if $P$ is isomorphic to $C_{p} \times C_{p}$, then $N_{G}(P)$ is a non-nilpotent group of order $p^{2} q$ isomorphic to the group described in the second part of Lemma 4.6: Suppose that $P \cong C_{p^{\alpha}}$ where $\alpha \geq 2$. The unique subgroup $C$ of the cyclic group $P$ of order $p$ must be normal in $N_{G}(P)$. Moreover, $P \neq N_{G}(P)$ by (I)-(II). It then follows from Lemma 4.5 that $N_{G}(P) / C$ is isomorphic to $C_{p r}$ and $\alpha=2$ where $r$ is a prime number different from $p$. So $N_{G}(P)$ is a non-abelian planar group of order $p^{2} r$ having a normal cyclic subgroup of order $p^{2}$, which is impossible by the virtue of Lemma 4.6. Consequently, it follows from the proof of (I) that $P$ is isomorphic to $C_{p} \times C_{p}$ or $C_{p}$. Suppose now that $P$ is isomorphic to $C_{p} \times C_{p}$. If $N_{G}(P) / P$ has a proper non-trivial subgroup $X / P$, then the set $\left\{N_{G}(P), X, P\right\}$ and the set consisting of any distinct three subgroups of $P$ of order $p$ form a $K_{3,3}$ in the intersection graph of $G$. Hence, $N_{G}(P) / P$ must have prime order $q$ so that $N_{G}(P)$ is a non-abelian planar group of order $p^{2} q$.

(IV) If $|G|$ is even, then any Sylow 2-subgroup of $G$ is isomorphic to $C_{2} \times C_{2}$ : Indeed, if there is a cyclic Sylow 2-subgroup $S$, then $S \cong C_{2}$ by (III). But then the N/C Lemma (see [9, p. 156]) implies that $N_{G}(S)=C_{G}(S)$ so that $S$ has a normal complement by the Burnside Normal Complement Theorem. 
(V) $G$ has no subgroup of order $2 s$ where $s$ is an odd prime: Suppose for a moment that $G$ has a subgroup $Y$ of order $2 s$. Let $U$ be a subgroup of $Y$ of order 2, and let $W$ be a Sylow 2-subgroup of $G$ containing $U$. From (IV) we know that $U \neq W$. Let $g$ be an element of $W$. Note that ${ }^{g} Y$ contains $U$. If $Y$ and ${ }^{g} Y$ are distinct, then the subgroups $U, W, N_{G}(W), Y,{ }^{g} Y$ form a $K_{5}$ in the intersection graph of $G$. Therefore, $Y={ }^{g} Y$ so that $g \in N_{G}(Y)$. Therefore, $W \leq N_{G}(Y)$, implying that $W Y=Y W$ so that $W Y$ is a subgroup of $G$ of order $4 s$. Note that $W Y \neq G$ (because $Y$ is a normal subgroup of $W Y$ ), and note that $W Y \neq N_{G}(W)$ (because otherwise $\left|N_{G}(W)\right|=2^{2} s$, and it follows from (III) and Lemma 4.6 that $N_{G}(W)$ has no subgroup of order $2 s$ ). Therefore, the subgroups $U, W, Y, W Y, N_{G}(W)$ form a $K_{5}$ in the intersection graph of $G$. This contradicts the planarity of $G$.

(VI) If $P$ is a Sylow $p$-subgroup of $G$ for some prime $p$ dividing $|G|$, then $P$ is isomorphic to $C_{p}$ : Assume contrarily that $P$ is a Sylow $p$-subgroup of $G$ not isomorphic to $C_{p}$. It follows from (III) that $P \cong C_{p} \times C_{p}$ and $N_{G}(P)$ is a non-abelian group of order $p^{2} q$ for some prime $q$ different from $p$. Let $Q$ be a Sylow $q$-subgroup of $N_{G}(P)$. It follows from (III) and Lemma 4.6 that $N_{G}(P) \cap N_{G}(Q)=Q$. Let $T$ be a Sylow $q$-subgroup of $G$ containing $Q$. If $T=Q$, then (II) implies that $Q \neq N_{G}(Q)$. If $T \neq Q$, then $N_{G}(Q)$ contains $T$ by (I) so that $Q \neq N_{G}(Q)$. Hence, $N_{G}(Q) / Q$ is a non-trivial group. For any two distinct elements $a Q$ and $b Q$ of the quotient group $N_{G}(Q) / Q$, the subgroups ${ }^{a} N_{G}(P)$ and ${ }^{b} N_{G}(P)$ are distinct subgroups containing $Q$ (because $N_{G}(P)$ is self normalizing and $\left.N_{G}(P) \cap N_{G}(Q)=Q\right)$. Therefore, if $\left|N_{G}(Q) / Q\right|>3$, then there are three distinct conjugates $Z_{1}, Z_{2}, Z_{3}$ of $N_{G}(P)$ containing $Q$, so that the subgroups $Z_{1}, Z_{2}, Z_{3}, Q, N_{G}(Q)$ form a $K_{5}$ in the intersection graph of $G$. Therefore, we must have that $\left|N_{G}(Q) / Q\right|=2$, and so $\left|N_{G}(Q)\right|=2 q$. But then, $(\mathrm{V})$ implies that $q=2$ and so $N_{G}(Q)$ is a Sylow 2-subgroup of $G$. Now the subgroups $N_{G}(P),{ }^{z} N_{G}(P), Q, N_{G}(Q), M$ form a $K_{5}$ in the intersection graph of $G$ where $z Q$ is any non-identity element of $N_{G}(Q) / Q$ and $M$ is the normalizer in $G$ of the Sylow 2-subgroup $N_{G}(Q)$ of $G$.

It follows from (VI) that $G$ has square free order. But such a group is solvable by Hölder's theorem (see Remark 1.1).

Proposition 5.2. A finite non-solvable group is not planar.

Proof. Suppose contrarily that $G$ is a finite non-solvable group which is planar. Since solvability is closed under group extension, $G$ must have a non-solvable simple composition factor $X$. It follows from Lemma 5.1 that $X$ is not isomorphic to a subgroup of $G$. Thus $X$ is isomorphic to $H / N$ for some non-trivial subgroup $H$ of $G$ and for some non-trivial proper normal subgroup $N$ of $H$. But then, as $H$ is planar, Lemma 4.5 implies that $X$ is abelian.

\section{References}

[1] J. Alonso, Groups of order $p q^{m}$ with elementary abelian Sylow $q$-subgroups, Proc. Amer. Math. Soc. 65 (1977), no. 1, 16-18. 
[2] J. P. Bohanon and L. Reid, Finite groups with planar subgroup lattices, J. Algebraic Combin. 23 (2006), no. 3, 207-223.

[3] W. Burnside, Theory of Groups of Finite Order, 2nd Edition, Dover Publications Inc., New York, 1955.

[4] F. N. Cole and J. W. Glover, On groups whose orders are products of three prime factors, Amer. J. Math. 15 (1893), no. 3, 191-220.

[5] D. Gorenstein, Finite Groups, 2nd Edition, Chelsea Publishing Co., New York, 1980.

[6] O. Hölder, Die Gruppen der Ordnungen $p^{3}, p q^{2}$, pqr, $p^{4}$, Math. Ann. 43 (1893), no. 2-3, 301-412.

[7] R. Le Vavasseur, Les groupes d'ordre $p^{2} q^{2}, p$ étant un nombre premier plus grand que le nombre premier q, Ann. Sci. École Norm. Sup. (3) 19 (1902), 335-355.

[8] G. A. Miller, Groups having a small number of subgroups, Proc. Nat. Acad. Sci. U.S.A. 25 (1939), 367-371.

[9] J. J. Rotman, An Introduction to the Theory of Groups, Fourth Edition, Graduate Texts in Mathematics, Vol. 148, Springer-Verlag, New York, 1995.

[10] R. Schmidt, On the occurrence of the complete graph $K_{5}$ in the Hasse graph of a finite group, Rend. Sem. Mat. Univ. Padova 115 (2006), 99-124.

[11] _ Planar subgroup lattices, Algebra Universalis 55 (2006), no. 1, 3-12.

[12] C. L. Starr and G. E. Turner, III, Planar groups, J. Algebraic Combin. 19 (2004), no. 3, 283-295.

[13] E. Yaraneri, Intersection graph of a module, J. Algebra Appl. 12 (2013), no. 5, 1250218, $30 \mathrm{pp}$.

SelÇUK Kayacan

Department of Mathematics

Istanbul Technical University

34469 Maslak, Istanbul, TURKey

E-mail address: skayacan@itu.edu.tr

ERGÜN YARANERI

Department of Mathematics

Istanbul Technical University

34469 Maslak, Istanbul, Turkey

E-mail address: eyaraneri@yahoo.com 